\title{
Marine Debris in Green Sea Turtles along the Northern Coast of Taiwan
}

\author{
I-Jiunn Cheng*, Pin-Chun Chou, Ying-Tin Chan and Tsung-Hsien Li \\ Institute of Marine Biology, National Taiwan Ocean University, Keelung, Taiwan, ROC, 202-24
}

*Corresponding author: I-Jiunn Cheng, Institute of Marine Biology, National

Taiwan Ocean University, Keelung, Taiwan.

Received Date: November 12,2020

Published Date: December 09,2020

\begin{abstract}
Sea turtles ingesting marine debris is a major problem worldwide. This is the first study ever done on the marine debris ingested by sea turtles in Taiwan, 191 dead green sea turtles from 2012 to 2019, mainly in northern coasts. We identified seven debris types and 15 colors. Plastic rope, soft and hard plastics, foam plastic and unidentified material were the most common ingested debris. White, transparent and mixture were either the common or the most common ingested color. Juveniles consumed more type of debris than the other age classes. Debris was found remain in the digestive tract for several weeks or longer prior to be found or excretion.
\end{abstract}

Keywords: Sea turtles; Marine debris; Plastic type; Plastic color; Digestive tract

\section{Introduction}

Marine debris is defined as any man-made solid waste product that is introduced into marine environment, and it has proven to have a widespread and negative impact on marine animals [1-4]. Most anthropogenic material ingested by living and dead individuals is made of plastics and pollutants [5]. The main sources of these debris are litter from ships, fishing and recreational boats, and garbage carried into the sea from land-based sources in industrialized and highly populated areas $[1,2,6]$. Coastal cities and towns are the main sources of plastic pollution, serving as point sources for the flow of plastic into the sea via urban and natural drainage systems [7]. Plastics are lightweight, strong, durable, and cheap, making them suitable materials for a wide range of products; they are ubiquitous on land, and make-up most of the marine litter worldwide [6,7]. Synthetic debris types include single-use plastics such as food packaging, fishing line and synthetic 'soft bait' lures [8]. Plastics are not biodegradable, but use in many shortlived disposable products, and have great dispersal capabilities, making them a severe global environmental threat to marine ecosystems [2,6,9]. Marine organisms may ingest plastic as a result of mistaken identity. As turtles are primarily visual feeders, they may misidentify items - such as shopping bags, and sheet plasticas prey and actively select them for consumption [3,10-12]. The presence of plastic in their foraging areas increases the likelihood that they accidentally ingest these materials $[12,13]$. Marine debris can cause mortality in sea turtles directly and indirectly. Sea turtles generally die from debris ingestion when their digestive tract is obstructed, and ingested plastic materials reduce their digestive capability, often resulting in emaciation and severe starvation [1316]. Gastrointestinal perforation caused by swallowed hooks and hard plastic can cause chronic infection, peritonitis, gastrointestinal motility disorders, and eventual death. $52 \%$ of surviving sea turtles have likely ingested marine debris, and a reported $4 \%$ of necropsied sea turtles have died from it [15]. The sub-lethal effects of anthropogenic debris on sea turtles are presumably more common than the lethal ones, but are difficult to measure because animals can excrete marine debris through defecation, which 
likely becomes a major threat in the long-term. The alimentary track of sea turtles is particularly prone to plication and debris accumulation due to the inability of turtles to regurgitate items and their convoluted gastrointestinal tracts [17]. Ingesting debris can be worsening the physical conditions, diminished food stimulus, blockage of gastric enzyme secretion, lowered steroid hormone levels, delayed ovulation, reproductive failure and death following blockage of the intestinal tract [2-4, 18-20]. Debris ingestion can create a false sense of fullness and interfere with lipid metabolism, resulting in a build-up of intestinal gases and associated buoyancy disorders (floating syndrome), thus limiting the turtle's ability to obtain food and avoid predation [4,21].Due to their hydrophobic properties, plastics are known to accumulate heavy metals and other toxins, such as PCBs, from the marine environment, which can also be released during digestion. These materials can alter hormonal processes, specifically those regulated by sex steroids $[1,2,11,22,23]$. Entanglement effects include abrasions, lesions, constriction, scoliosis, or the loss of limbs, as well as increased drag, which may reduce foraging efficiency and avoidance ability [7]. There are many studies conduct on plastic ingestion of different species, gender, growth stage, etc. $[15,17]$. However, it is important to compare the plastic ingestion between species, sexes, and growth stages of sea turtles in the same region, because different status and species sea turtles may come from different sites and may select ingest different type of debris. Then, one can determine if and how the plastics are ingested differently based on the different species or status. Then, the proper recommendations can be made.

\section{Material and Methods}

\section{Sample collection}

Samples were mainly collected from Hualien County in eastern Taiwan to Taichung County in western Taiwan from 2012 to 2019 (Figure 1)

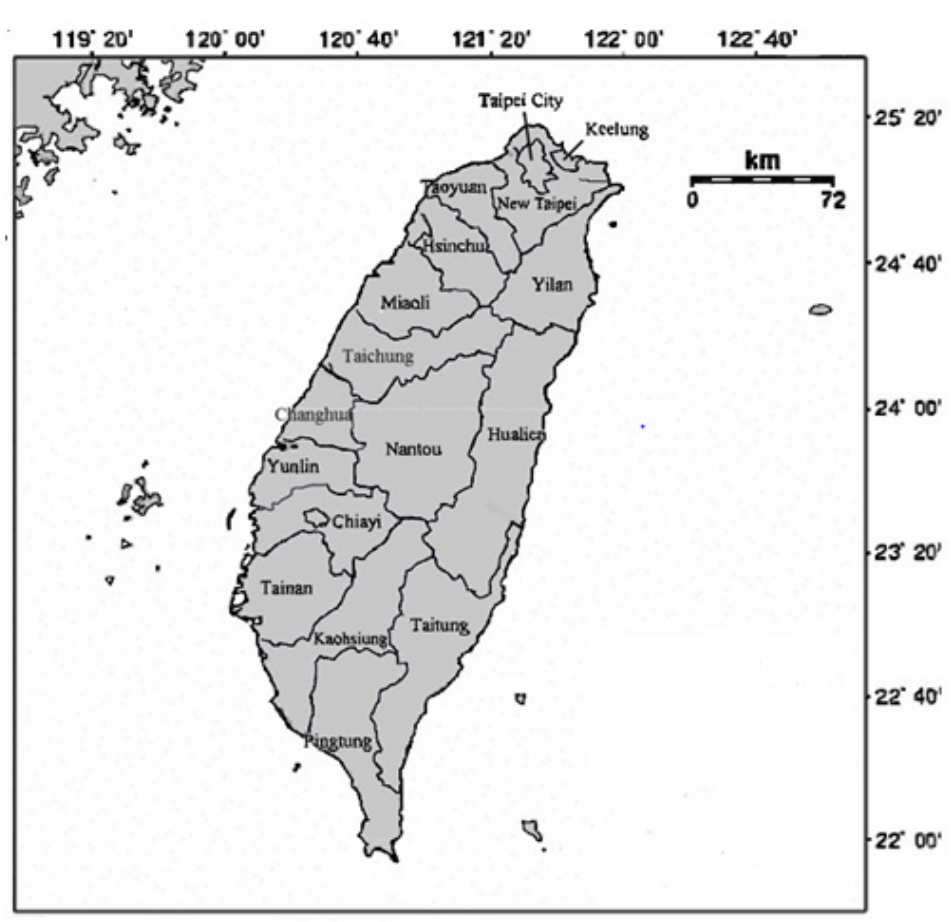

Figure 1: Map of Taiwan with county name of counties appear in the graph.

A total of 191 turtles were collected. Body length and body weight were measured prior to necropsy. Body length was expressed as curved carapace length (CCL), measured from the tip to the notch of the dorsal carapace. Necropsy was carried out on turtles that were either stranded dead or rescued but did not survive during rehabilitation. The entire digestive tract-including the esophagus, stomach, and intestines-was removed for analyses. Gender was determined during necropsy by visually examining the morphology of the gonad [24]. Age class was determined based on body size; CCL $<35 \mathrm{~cm}$ was considered hatchling, $35<\mathrm{CCL} \leq 55$ was juvenile, $55<\mathrm{CCL} \leq 90$ was sub adult, and $>90$ was adult.

\section{Sample treatment}

First, the gross morphology was observed to determine the body conditions and if the turtle was entangled by foreign materials. Necropsy was performed following the procedure of [24]. Samples were removed from analysis if their digestive tract was decayed beyond recognition, or was fractured during the sampling procedure. Intact digestive tracts were brought back to the laboratory and stored in a refrigerator for later analysis. In the laboratory, the digestive tract was cut open and the contents of the esophagus, stomach, and intestines were washed with tap water and filtered through a $0.05-\mathrm{cm}$ mesh filter. Marine debris 
was collected from the filter and dried at room temperature. Debris from each digestive tract section was stored in plastic bags. The different parts of the digestive tract were separated following [2]. The esophagus stretches from the anterior end of the digestive tract to the gastroesophageal sphincter. The stomach is found from gastroesophageal sphincter to the constriction duodenal. The intestine is found from constriction duodenal to the end of the digestive tract. The digestive tract is further divided into five sections: sections 1 and 2 refer to the small intestine, and sections 3 to 5 refer to the large intestine.

\section{Identifying marine debris}

Marine debris was identified visually, with a stereomicroscope if necessary. Debris was separated based on material type (soft plastic, hard plastic, cotton thread, plastic rope, foam plastic, textile, rubber, metal, or unidentified) and color (transparent, semi-transparent, white, black, grey, silver, green, brown, orange, yellow, purple, blue, green pink, red, or mixture). Soft plastic was defined as a deformable non-thread plastic material, like straws or plastic bags. Hard plastic was defined as a non-deformable nonthread plastic material, like fragments of table ware or plastic cups. Plastic rope was defined as thread-like plastic material, like fishing line or fishing nets. Foam plastic was defined as foaming plastic, including artificial sponges or crash cushions. Textile included cloth. Rubber included parts of tile. Metal included fishing hooks. Unidentified material was defined as any material that was decayed beyond recognition. Color was determinate visually. In addition to anthropogenic materials, natural debris were also collected and identified, i.e. wood (branches, wood products). All materials were counted and weighed (to $0.001 \mathrm{mg}$ ) and expressed as a quantity, weight, and abundance (percentage of its weight to the total of all debris found). To avoid repeating calculations, only particles larger than $0.5 \mathrm{~cm}$ long were included. This is because most material smaller than $0.5 \mathrm{~cm}$ long is a fragment of medium to large debris in the digestive tract. In order to determine the frequency at which each consumed debris item occurred, the frequency of occurrence (FO) was calculated as FO $(\%)=(\mathrm{Ni} / \mathrm{Nt}) * 100$, where $\mathrm{Ni}$ is the abundance or weight of item $\mathrm{i}$ and $\mathrm{Nt}$ is the total abundance or weight of all debris. FO was separated into three categories: $>50 \%$ : the most common, $50 \%<$ FO $\leq 10 \%$ : common, and $<10 \%$ : uncommon [25]. Debris that weighed too little to measure were not included in the FO analysis. The index of relative importance (IRI) was determined to measure the relative impact that each item had on the turtle's health. It was calculated as IRI of species $=(\%$ occurrence * \% weight)/100 [26]. We separated the IRI into three categories based on von Brandis et al. [26]: $30<$ IRI $\leq 100$ : the most important, $30<\mathrm{IRI} \leq 10$ : important, and $<10$ : unimportant. The Body Condition Index (BCI) of each species was calculated to determine the health status of turtles:

$\mathrm{BCI}=($ turtle body mass $/$ SCL3) $* 1000$ [27].

The body burden (BB) of each species was calculated to determine the impact that each ingested debris had on the body condition:

$\mathrm{BB}=\mathrm{mg}$ debris weight ingested $/ \mathrm{kg}$ turtle weight [28].

\section{Statistical analyses}

The Shapiro-Wilk test was used to determine if the marine debris showed a normal distribution. If not, the Kruskal-Wallis test was used to compare the differences among material types and colors of marine debris [29]. One-way Analysis of Variance (ANOVA) was used to determine whether the samples had a normal distribution. Turkey's post-hoc test was used to determine the differences among variables [29]. The Pearson correlation test was used to determine if the body burden was correlated with the body size (straight carapace length; SCL), and if the body condition (BCI) was correlated with the abundance of ingested debris.

\section{Result}

\section{Total debris ingestion}

Eighty-six percentage of the stranded turtles ingested marine debris. Analysis showed that the hard and soft plastics, plastic rope, foam plastic and unidentified material were the common while unimportant ingested debris (Table 1).

Table 1: Number and weight-specific debris ingested, FO (\%) and IRI of different debris type ingested by green turtles. Unit: mg in weight.

\begin{tabular}{|c|c|c|c|c|c|c|c|c|c|c|}
\hline Parameter & Soft Plastic & Hard Plastic & $\begin{array}{l}\text { Cotton } \\
\text { Thread }\end{array}$ & Plastic Rope & Foam Plastic & Textile & Rubber & Wood & Metal & $\begin{array}{l}\text { Unidenti- } \\
\text { fied }\end{array}$ \\
\hline Number & $21 \pm 41(98)$ & $34 \pm 68(64)$ & $6 \pm 7(32)$ & $18 \pm 19(150)$ & $24 \pm 38(71)$ & $10 \pm 27(22)$ & $2 \pm 2(16)$ & $10 \pm 21(10)$ & $1 \pm 0(8)$ & $24 \pm 44(44)$ \\
\hline Weight & $6.3 \pm 19(98)$ & $9.1 \pm 22.3(64)$ & $1.8 \pm 2.4(32)$ & $3.5 \pm 7.2(150)$ & $4.1 \pm 8.9(71)$ & $0.4 \pm 0.5(22)$ & $2.3 \pm 2.7(16)$ & $2.2 \pm 2.4(10)$ & $0.7 \pm 0.6(10)$ & $3.2 \pm 5.1(44)$ \\
\hline FO & 20 & 21 & 2 & 26 & 17 & 2 & $<1$ & 1 & $<1$ & 11 \\
\hline IRI & 6 & 6 & $<1$ & 6 & 2 & $<1$ & $<1$ & $<1$ & $<1$ & $<1$ \\
\hline
\end{tabular}

Statistical analysis showed that the turtles ingested more soft, hard plastics and plastic rope than the metal, cotton thread, and rubber. They also ingested more foam plastic than rubber, metal, cotton thread, and textile. Turtles ingested more unidentified material than rubber or metal ( $p<0.001$ in all cases). In term of debris weight, green turtles ingested more hard plastic than textile or wood $(\mathrm{p}<0.001)$. For the color debris, white was the common and most important ingested color, transparent was the common and important ingested color, and mixture was the important ingested color (Table 2). 
Table 2: Number-specific and FO (\%) of ingested different color debris by green turtles.

\begin{tabular}{|c|c|c|c|c|c|c|c|c|c|c|c|c|c|c|c|}
\hline $\begin{array}{l}\text { Pa- } \\
\text { rame- } \\
\text { ter }\end{array}$ & $\begin{array}{l}\text { Trans- } \\
\text { parent }\end{array}$ & $\begin{array}{l}\text { Semi- } \\
\text { Transpar- } \\
\text { ent }\end{array}$ & White & Black & Grey & Silver & Brown & Orange & Yellow & Purple & Blue & Green & Pink & Red & Mixture \\
\hline $\begin{array}{l}\text { Num- } \\
\text { ber }\end{array}$ & $\begin{array}{l}17 \pm 13 \\
(109)\end{array}$ & $\begin{array}{l}5 \pm 6 \\
(32)\end{array}$ & $\begin{array}{r}32 \pm 67 \\
(126)\end{array}$ & $\begin{array}{l}6 \pm 8 \\
(91)\end{array}$ & $\begin{array}{l}3 \pm 4 \\
(47)\end{array}$ & 1 & $\begin{array}{l}7 \pm 18 \\
(20)\end{array}$ & $\begin{array}{l}3 \pm 3 \\
(18)\end{array}$ & $\begin{array}{l}4 \pm 4 \\
(69)\end{array}$ & $1 \pm 1(11)$ & $3 \pm 3(57)$ & $6 \pm 8(109)$ & $4 \pm 4(32)$ & $4 \pm 4(32)$ & $4 \pm 4(32)$ \\
\hline FO & 19 & 2 & 44 & 5 & 1 & 0 & 2 & 1 & 3 & 0 & 2 & 7 & 1 & 2 & 12 \\
\hline
\end{tabular}

\section{Debris ingestion among different sex}

Forty-eight males, 90 females and 53 unknown sex were recorded. The study found that for all genders, soft plastic and plastic rope were the common and either important or unimportant ingested debris. Hard plastic and foam plastic were the common while unimportant ingested debris of male and unknown sex turtles (Table 3).

Statistical analysis showed that male turtles ingested less rubber and cotton thread than the other debris $(\mathrm{p}<0.001)$. In term of debris weight, male turtle ingested more hard and soft plastics than the cotton thread. They also ingested more hard plastic than plastic rope $(p<0.001)$. Unknown sex turtles ingested more foam plastic than metal $(p=0.001)$. For all genders, transparent was the common ingested color. Mixture was the common ingested color of male and unknown sex turtles, and white was the common ingested color of the female and unknown sex turtles (Table 4).
Statistical analysis showed that the turtles ingested more white debris than purple and red, and ingested more transparent than purple debris $(\mathrm{p}<0.001)$. Comparison among different genders showed that male turtle ingested unidentified debris than the female turtles ( $\mathrm{p}=0.013)$.

\section{Debris ingestion among different age class}

Three hatchlings, 143 juveniles, 37 sub adults and 8 adults were recorded. The study found that the soft plastic was the common while unimportant ingested debris of hatchling, juvenile and adult turtles. Plastic rope was either the common or most common and either important or unimportant ingested debris of juvenile, sub adult and adult turtles. Hard plastic was either common or most common and either important or unimportant ingested debris of both hatchling and juvenile turtles. Foam plastic was common while unimportant ingested debris of both juvenile and sub adult turtles. Unidentified material was the common while unimportant ingested debris of both juvenile and adult turtles (Table 5).

Table 3: Number of turtles, number- and weight-specific, FO (\%) and IRI of each ingested debris type of different gender of green turtles. Unit is mg for weight.

\begin{tabular}{|c|c|c|c|c|c|c|c|c|c|c|c|c|}
\hline Gender & Turtles & Parameter & Soft Plastic & $\begin{array}{l}\text { Hard } \\
\text { Plastic }\end{array}$ & $\begin{array}{l}\text { Cotton } \\
\text { Thread }\end{array}$ & $\begin{array}{l}\text { Plastic } \\
\text { Rope }\end{array}$ & $\begin{array}{l}\text { Foam } \\
\text { Plastic }\end{array}$ & Textile & Rubber & Wood & Metal & $\begin{array}{l}\text { Unidenti- } \\
\text { fied }\end{array}$ \\
\hline \multirow{4}{*}{ Male } & \multirow{4}{*}{48} & number & $\begin{array}{c}23 \pm 20 \\
(14)\end{array}$ & $\begin{array}{c}17 \pm 22 \\
(10)\end{array}$ & 0 & $9 \pm 11(24)$ & $12 \pm 9(9)$ & 0 & $\begin{array}{l}1 \pm 0 \\
(5)\end{array}$ & 0 & 0 & $4 \pm 3(3)$ \\
\hline & & weight & $1.4 \pm 2(14)$ & $\begin{array}{c}0.8 \pm 0.9 \\
(10)\end{array}$ & 0 & 0.1 & $0.2 \pm 0.2(9)$ & 0 & $\begin{array}{c}0.2 \pm 0.1 \\
(5)\end{array}$ & 0 & 0 & $0.2 \pm 0.1(3)$ \\
\hline & & FO & 32 & 23 & 0 & 29 & 12 & 0 & 1 & 0 & 0 & 2 \\
\hline & & IRI & 19 & 7 & 0 & $<1$ & 1 & 0 & $<1$ & 0 & 0 & $<1$ \\
\hline \multirow{4}{*}{ Female } & \multirow{4}{*}{90} & number & $15 \pm 37(42)$ & $\begin{array}{c}27 \pm 69 \\
(22)\end{array}$ & $7 \pm 8(23)$ & $21 \pm 56(76)$ & $\begin{array}{c}22 \pm 41 \\
(33)\end{array}$ & $\begin{array}{c}17 \pm 39 \\
(11)\end{array}$ & $\begin{array}{l}2 \pm 2 \\
(9)\end{array}$ & $\begin{array}{l}3 \pm 5 \\
(4)\end{array}$ & $1 \pm 0(3)$ & $13 \pm 24(23)$ \\
\hline & & weight & $8.6 \pm 6.2(42)$ & $\begin{array}{c}4.7 \pm 8.3 \\
(22)\end{array}$ & $\begin{array}{c}2.3 \pm 2.7 \\
(23)\end{array}$ & $4.6 \pm 8(76)$ & $\begin{array}{c}5.7 \pm 12.7 \\
(33)\end{array}$ & $\begin{array}{c}0.5 \pm 0.7 \\
(11)\end{array}$ & $\begin{array}{c}1.8 \pm 1.9 \\
(9)\end{array}$ & $0.2 \pm 0.2(4)$ & $0.6 \pm 0.9(3)$ & $4 \pm 6.2(23)$ \\
\hline & & FO & 15 & 15 & 4 & 38 & 17 & 4 & 1 & $<1$ & $<1$ & 7 \\
\hline & & IRI & 5 & 1 & $<1$ & 12 & 3 & $<1$ & $<1$ & $<1$ & $<1$ & $<1$ \\
\hline \multirow{4}{*}{$\begin{array}{c}\text { Un- } \\
\text { known } \\
\text { Sex }\end{array}$} & \multirow{4}{*}{5} & number & $\begin{array}{c}27 \pm 49 \\
(38)\end{array}$ & $\begin{array}{c}40 \pm 89 \\
(24)\end{array}$ & 0 & $15 \pm 22(39)$ & $\begin{array}{c}29 \pm 41 \\
(24)\end{array}$ & $\begin{array}{l}4 \pm 4 \\
(12)\end{array}$ & 0 & $\begin{array}{c}14 \pm 28 \\
(6)\end{array}$ & $1 \pm 0(4)$ & $16 \pm 22(8)$ \\
\hline & & weight & $\begin{array}{l}1.6 \pm 3 \\
(38) \\
\end{array}$ & $\begin{array}{c}2.3 \pm 4.6 \\
(24)\end{array}$ & 0 & $0.8 \pm 1(39)$ & $\begin{array}{c}1.3 \pm 1.7 \\
(24) \\
\end{array}$ & $\begin{array}{c}0.3 \pm 0.4 \\
(12) \\
\end{array}$ & 0 & $0.3 \pm 0.5(6)$ & $0.7 \pm 0.4(4)$ & $0.2 \pm 0.2(8)$ \\
\hline & & FO & 29 & 28 & 0 & 16 & 20 & 1 & 0 & 2 & $<1$ & 4 \\
\hline & & IRI & 9 & 8 & 0 & 3 & 3 & $<1$ & 0 & $<1$ & $<1$ & $<1$ \\
\hline
\end{tabular}


Table 4: Number of turtles, number-specific and FO (\%) of ingested different color debris by different genders of green turtles.

\begin{tabular}{|c|c|c|c|c|c|c|c|c|c|c|c|c|c|c|c|c|c|}
\hline Gender & $\begin{array}{c}\text { Tur- } \\
\text { tle }\end{array}$ & $\begin{array}{l}\text { Pa- } \\
\text { ram- } \\
\text { eter }\end{array}$ & $\begin{array}{l}\text { Trans- } \\
\text { parent }\end{array}$ & $\begin{array}{c}\text { Semi- } \\
\text { Transpa } \\
\text { rent }\end{array}$ & White & Black & Grey & $\begin{array}{l}\text { Sil- } \\
\text { ver }\end{array}$ & $\begin{array}{l}\text { Bro } \\
\text { wn }\end{array}$ & $\begin{array}{l}\text { Or- } \\
\text { ange }\end{array}$ & $\begin{array}{l}\text { Yel- } \\
\text { low }\end{array}$ & $\begin{array}{c}\text { Pur- } \\
\text { ple }\end{array}$ & Blue & Green & Pink & Red & $\begin{array}{l}\text { Mix- } \\
\text { ture }\end{array}$ \\
\hline \multirow[t]{2}{*}{ Male } & \multirow[t]{2}{*}{48} & $\begin{array}{l}\text { num- } \\
\text { ber }\end{array}$ & $\begin{array}{c}14 \pm 18 \\
(24)\end{array}$ & $\begin{array}{l}9 \pm 7 \\
(12)\end{array}$ & $\begin{array}{l}5 \pm 4 \\
(20)\end{array}$ & $\begin{array}{l}5 \pm 4 \\
(20)\end{array}$ & $\begin{array}{l}3 \pm 3 \\
(17)\end{array}$ & 0 & $\begin{array}{l}3 \pm 1 \\
(2)\end{array}$ & $1 \pm 1(5)$ & $\begin{array}{l}4 \pm 4 \\
(16)\end{array}$ & 1 & $\begin{array}{l}4 \pm 4 \\
(16)\end{array}$ & $\begin{array}{l}5 \pm 4 \\
(27)\end{array}$ & $\begin{array}{l}5 \pm 5 \\
(12)\end{array}$ & $\begin{array}{l}3 \pm 2 \\
(8)\end{array}$ & $\begin{array}{c}16 \pm 27 \\
(32)\end{array}$ \\
\hline & & FO & 22 & 7 & 6 & 6 & 3 & 0 & $<1$ & $<1$ & 4 & $<1$ & 4 & 9 & 4 & 2 & 33 \\
\hline \multirow[t]{2}{*}{ Female } & \multirow[t]{2}{*}{90} & $\begin{array}{l}\text { num- } \\
\text { ber }\end{array}$ & $\begin{array}{c}12 \pm 28 \\
(53)\end{array}$ & $\begin{array}{l}3 \pm 3 \\
(18)\end{array}$ & $\begin{array}{c}24 \pm 62 \\
(68)\end{array}$ & $\begin{array}{l}6 \pm 10 \\
(39)\end{array}$ & $\begin{array}{l}2 \pm 2 \\
(20)\end{array}$ & 1 & $\begin{array}{l}4 \pm 5 \\
(5)\end{array}$ & $2 \pm 1(7)$ & $\begin{array}{l}3 \pm 2 \\
(31)\end{array}$ & $\begin{array}{l}2 \pm 1 \\
(5)\end{array}$ & $\begin{array}{l}3 \pm 3 \\
(27) \\
\end{array}$ & $\begin{array}{l}6 \pm 9 \\
(52)\end{array}$ & $\begin{array}{l}3 \pm 4 \\
(16)\end{array}$ & $\begin{array}{l}3 \pm 3 \\
(23)\end{array}$ & $\begin{array}{c}11 \pm 19 \\
(49)\end{array}$ \\
\hline & & FO & 17 & 1 & 44 & 6 & 1 & $<1$ & 1 & $<1$ & 2 & $<1$ & 2 & 8 & 1 & 2 & 14 \\
\hline \multirow[t]{2}{*}{$\begin{array}{l}\text { Unknown } \\
\text { Sex }\end{array}$} & \multirow[t]{2}{*}{5} & $\begin{array}{l}\text { num- } \\
\text { ber }\end{array}$ & $\begin{array}{c}26 \pm 45 \\
(32)\end{array}$ & 0 & $\begin{array}{c}44 \pm 70 \\
(34)\end{array}$ & $\begin{array}{l}6 \pm 8 \\
(32)\end{array}$ & $\begin{array}{l}5 \pm 6 \\
(9)\end{array}$ & 0 & $\begin{array}{l}9 \pm 22 \\
(13)\end{array}$ & $6 \pm 5(6)$ & $\begin{array}{l}5 \pm 6 \\
(19)\end{array}$ & $\begin{array}{l}1 \pm 0 \\
(5)\end{array}$ & $\begin{array}{l}4 \pm 3 \\
(16 .)\end{array}$ & $\begin{array}{l}6 \pm 7 \\
(30)\end{array}$ & $\begin{array}{l}5 \pm 1 \\
(2)\end{array}$ & $\begin{array}{l}4 \pm 5 \\
(17)\end{array}$ & $\begin{array}{c}6 \pm 14 \\
(9)\end{array}$ \\
\hline & & FO & 25 & 0 & 51 & 5 & 1 & 0 & 3 & 1 & 3 & $<1$ & 2 & 5 & 0 & 2 & 2 \\
\hline
\end{tabular}

Table 5: Number of turtles, number- and weight-specific, FO (\%) and IRI of ingested different debris type of different age class of green turtles. Unit: mg in weight.

\begin{tabular}{|c|c|c|c|c|c|c|c|c|c|c|c|c|}
\hline $\begin{array}{l}\text { Age } \\
\text { Class }\end{array}$ & $\begin{array}{l}\text { Tur- } \\
\text { tles }\end{array}$ & $\begin{array}{l}\text { Pa- } \\
\text { rame- } \\
\text { ter }\end{array}$ & $\begin{array}{c}\text { Soft Plas- } \\
\text { tic }\end{array}$ & $\begin{array}{l}\text { Hard } \\
\text { Plastic }\end{array}$ & $\begin{array}{l}\text { Cotton } \\
\text { Thread }\end{array}$ & $\begin{array}{c}\text { Plastic } \\
\text { Rope }\end{array}$ & $\begin{array}{c}\text { Foam Plas- } \\
\text { tic }\end{array}$ & Textile & Rubber & Wood & Metal & Unidentified \\
\hline \multirow{4}{*}{$\begin{array}{l}\text { Hatch- } \\
\text { ling }\end{array}$} & \multirow{4}{*}{3} & $\begin{array}{l}\text { num- } \\
\text { ber }\end{array}$ & $\begin{array}{l}5 \pm 4 \\
(2)\end{array}$ & $\begin{array}{c}25 \pm 33 \\
(2) \\
\end{array}$ & 0 & 0 & 0 & 0 & 0 & 0 & 0 & 0 \\
\hline & & weight & 0.2 & $\begin{array}{c}0.3 \pm 0.3 \\
(2)\end{array}$ & 0 & 0 & 0 & 0 & 0 & 0 & 0 & 0 \\
\hline & & FO & 16 & 84 & 0 & 0 & 0 & 0 & 0 & 0 & 0 & 0 \\
\hline & & IRI & 6 & 52 & 0 & 0 & 0 & 0 & 0 & 0 & 0 & 0 \\
\hline \multirow{4}{*}{ Juvenile } & \multirow{4}{*}{145} & $\begin{array}{l}\text { num- } \\
\text { ber }\end{array}$ & $\begin{array}{c}25 \pm 45 \\
(75) \\
\end{array}$ & $\begin{array}{c}39 \pm 73 \\
(58) \\
\end{array}$ & 0 & $\begin{array}{l}14 \pm 19 \\
(111)\end{array}$ & $\begin{array}{c}23 \pm 33 \\
(58) \\
\end{array}$ & $4 \pm 4(16)$ & $2 \pm 2(12)$ & $11 \pm 22(9)$ & $1 \pm 0(7)$ & $28 \pm 51(29)$ \\
\hline & & weight & $\begin{array}{c}5.1 \pm 12 \\
(75)\end{array}$ & $\begin{array}{c}10.7 \pm 24.5 \\
(58)\end{array}$ & 0 & $\begin{array}{c}2.9 \pm 6.2 \\
(111)\end{array}$ & $\begin{array}{c}2.8 \pm 4.7 \\
(58)\end{array}$ & $0.3 \pm 0.4(16)$ & $2.7 \pm 3(12)$ & $0.3 \pm 0.4(9)$ & $0.5 \pm 0.4(7)$ & $3.2 \pm 3.6(29)$ \\
\hline & & FO & 24 & 26 & 0 & 19 & 17 & 1 & $<1$ & 1 & $<1$ & 10 \\
\hline & & IRI & 6 & 9 & 0 & 4 & 2 & $<1$ & $<1$ & $<1$ & $<1$ & $<1$ \\
\hline \multirow{4}{*}{$\begin{array}{l}\text { Sub- } \\
\text { adult }\end{array}$} & \multirow{4}{*}{37} & $\begin{array}{l}\text { num- } \\
\text { ber }\end{array}$ & $\begin{array}{l}9 \pm 14 \\
(19)\end{array}$ & $\begin{array}{c}8 \pm 13 \\
(7) \\
\end{array}$ & 0 & $\begin{array}{c}33 \pm 77 \\
(31) \\
\end{array}$ & $\begin{array}{c}33 \pm 62 \\
(10) \\
\end{array}$ & $25 \pm 51(6)$ & $2 \pm 1(2)$ & 0 & 1 & $18 \pm 29(10)$ \\
\hline & & weight & $\begin{array}{c}12.7 \pm 26.6 \\
(19)\end{array}$ & $\begin{array}{c}3.7 \pm 4.6 \\
(7)\end{array}$ & 0 & $\begin{array}{l}4.5 \pm 9 \\
(31) \\
\end{array}$ & $\begin{array}{c}11.9 \pm 19.1 \\
(10)\end{array}$ & $0.5 \pm 0.9(6)$ & 0.3 & 0 & 2 & $0.2 \pm 9.3(5)$ \\
\hline & & FO & 8 & 3 & 0 & 51 & 17 & 8 & $<1$ & 0 & $<1$ & 9 \\
\hline & & IRI & 3 & $<1$ & 0 & 12 & 4 & $<1$ & $<1$ & 0 & $<1$ & $<1$ \\
\hline \multirow{4}{*}{ Adult } & \multirow{4}{*}{8} & $\begin{array}{l}\text { num- } \\
\text { ber }\end{array}$ & $\begin{array}{c}15 \pm 29 \\
(4) \\
\end{array}$ & $\begin{array}{c}16 \pm 16 \\
(2) \\
\end{array}$ & $\begin{array}{l}2 \pm 2 \\
(3)\end{array}$ & $\begin{array}{c}13 \pm 21 \\
(8) \\
\end{array}$ & $\begin{array}{l}8 \pm 0 \\
(3) \\
\end{array}$ & 0 & $1 \pm 0(2)$ & 0 & 1 & $16 \pm 29(5)$ \\
\hline & & weight & $\begin{array}{c}1.6 \pm 1.9 \\
(4)\end{array}$ & $\begin{array}{c}0.9 \pm 1.3 \\
(2)\end{array}$ & $\begin{array}{c}0.6 \pm 0.9 \\
(3)\end{array}$ & $\begin{array}{c}8 \pm 11 \\
(7)\end{array}$ & $\begin{array}{c}1.2 \pm 0.6 \\
(3)\end{array}$ & 0 & $1.1 \pm 1(2)$ & 0 & 0.2 & $0.3 \pm 0.2(5)$ \\
\hline & & FO & 20 & 8 & 2 & 34 & 8 & 0 & 1 & 0 & $<1$ & 26 \\
\hline & & IRI & 2 & $<1$ & $<1$ & 26 & $<1$ & 0 & $<1$ & 0 & $<1$ & $<1$ \\
\hline
\end{tabular}

Statistical analysis showed that the juvenile turtles ingested more hard plastic and foam plastic than the metal, rubber, textile and cotton thread. They also ingested more soft plastic than the rubber and textile $(\mathrm{p}<0.001)$. Compare among different age classes showed that juvenile ingested more types of debris than the other age classes. Statistical analysis showed that juvenile ingested more pieces of debris than adult turtle $(p=0.039)$. In term of ingested color, white was either the common or most common ingested 
color of all age classes. Transparent and mixture were the common ingested color of juvenile, sub adult and adult turtles (Table 6).

\section{BCI and BB of all species and the relationship with the body length (SCL) and ingested debris abundance}

The number of either SCL or debris ingestion of both hatchling and adult turtles were less than 2, these two parameters were excluded from analyses. The mean BCI ranged from 0.09 (male and subadult) to 1.84 (juvenile), and mean BB ranged from 0.65 (unknown sex) to 4.23 (male) (Table 7).

Analysis showed that the BCI of the genders and age classes were not influenced by the ingested debris weight $(p=0.57$ for male turtles, $p=0.982$ for female turtles, $p=0.393$ for unknown sex turtles, $p=0.962$ for juvenile turtles, $p=0.626$ for subadult turtles). Except female turtle, BB was also not influenced by the ingested debris abundance $(\mathrm{p}=0.63$ for male turtles, $\mathrm{p}=0.578$ for unknown sex turtles, $p=0.175$ for juvenile turtles, $p=0.903$ for subadult turtles). For the female turtle, body burden (BB) was found increased with the straight carapace length of the turtles (BB $=-2.921+0.0899(\mathrm{SCL}), \mathrm{n}=63, \mathrm{r}=0.276, \mathrm{p}=0.029$ ).

\section{Proportion of debris in each section of the intestine tract}

(Table 8) Fifty-night turtles were available for determining the position of debris within the digestive tracts. A total of 100 pieces of debris were collected. Analyzed showed the debris contained in each section of digestive tract were $2 \%$ in the esophagus, $5 \%$ in the stomach, $2 \%$ in stomach I, $13 \%$ in stomach II, $23 \%$ in stomach III, $32 \%$ in stomach IV and $19 \%$ in stomach V.

Table 6: Number of turtles, number-specific and FO (\%) of ingested different color by different age class of green turtles.

\begin{tabular}{|c|c|c|c|c|c|c|c|c|c|c|c|c|c|c|c|c|c|}
\hline $\begin{array}{l}\text { Tur- } \\
\text { tles }\end{array}$ & $\begin{array}{l}\text { Age } \\
\text { Class }\end{array}$ & $\begin{array}{l}\text { Pa- } \\
\text { ram- } \\
\text { eter }\end{array}$ & $\begin{array}{l}\text { Trans- } \\
\text { parent }\end{array}$ & $\begin{array}{l}\text { Semi- } \\
\text { Trans- } \\
\text { pa } \\
\text { rent }\end{array}$ & White & Black & Grey & $\begin{array}{l}\text { Sil- } \\
\text { ver }\end{array}$ & Brown & $\begin{array}{l}\text { Or- } \\
\text { ange }\end{array}$ & $\begin{array}{l}\text { Yel- } \\
\text { low }\end{array}$ & $\begin{array}{c}\text { Pur- } \\
\text { ple }\end{array}$ & Blue & Green & Pink & Red & $\begin{array}{l}\text { Mix- } \\
\text { ture }\end{array}$ \\
\hline \multirow[t]{2}{*}{3} & \multirow[t]{2}{*}{$\begin{array}{l}\text { hatch- } \\
\text { ling }\end{array}$} & $\begin{array}{l}\text { num- } \\
\text { ber }\end{array}$ & 5 & 5 & $\begin{array}{c}24 \pm 30 \\
(2)\end{array}$ & 1 & 0 & 0 & 0 & 0 & 0 & 0 & 2 & 1 & 0 & 2 & 0 \\
\hline & & FO & 9 & $<1$ & 81 & 2 & 0 & 0 & 0 & 0 & 0 & 0 & 3 & 2 & 0 & 3 & 0 \\
\hline \multirow[t]{2}{*}{145} & \multirow[t]{2}{*}{$\begin{array}{l}\text { juve- } \\
\text { nile }\end{array}$} & $\begin{array}{l}\text { num- } \\
\text { ber }\end{array}$ & $\begin{array}{c}19 \pm 37 \\
(81)\end{array}$ & $\begin{array}{l}6 \pm 7 \\
(22)\end{array}$ & $\begin{array}{l}30 \pm 57 \\
(103)\end{array}$ & $\begin{array}{l}5 \pm 6 \\
(72)\end{array}$ & $\begin{array}{l}3 \pm 4 \\
(33)\end{array}$ & 1 & $\begin{array}{l}9 \pm 20 \\
(15)\end{array}$ & $\begin{array}{l}3 \pm 4 \\
(17)\end{array}$ & $\begin{array}{l}4 \pm 4 \\
(53)\end{array}$ & $\begin{array}{l}1 \pm 1 \\
(8)\end{array}$ & $\begin{array}{l}4 \pm 4 \\
(40)\end{array}$ & $\begin{array}{l}5 \pm 6 \\
(79)\end{array}$ & $\begin{array}{l}4 \pm 4 \\
(24)\end{array}$ & $3 \pm 3(38)$ & $\begin{array}{c}14 \pm 25 \\
(61)\end{array}$ \\
\hline & & FO & 21 & 2 & 42 & 5 & 2 & $<1$ & 2 & 1 & 3 & $<1$ & 2 & 6 & 1 & 2 & 12 \\
\hline \multirow[t]{2}{*}{37} & \multirow[t]{2}{*}{$\begin{array}{l}\text { sub- } \\
\text { adult }\end{array}$} & $\begin{array}{l}\text { num- } \\
\text { ber }\end{array}$ & $\begin{array}{l}8 \pm 10 \\
(23) \\
\end{array}$ & $\begin{array}{l}4 \pm 3 \\
(9)\end{array}$ & $\begin{array}{c}43 \pm 102 \\
(21)\end{array}$ & $\begin{array}{l}9 \pm 15 \\
(14) \\
\end{array}$ & $\begin{array}{l}2 \pm 2 \\
(11) \\
\end{array}$ & 1 & $\begin{array}{l}3 \pm 1 \\
(4)\end{array}$ & 3 & $\begin{array}{l}3 \pm 2 \\
(9)\end{array}$ & $\begin{array}{l}1 \pm 0 \\
(2)\end{array}$ & $\begin{array}{l}3 \pm 4 \\
(13) \\
\end{array}$ & $\begin{array}{l}8 \pm 12 \\
(24)\end{array}$ & $\begin{array}{l}2 \pm 2 \\
(3)\end{array}$ & $5 \pm 5(6)$ & $\begin{array}{l}9 \pm 11 \\
(23)\end{array}$ \\
\hline & & FO & 11 & 2 & 50 & 7 & 1 & $<1$ & 1 & $<1$ & 2 & 0 & 2 & 10 & $<1$ & 2 & 12 \\
\hline \multirow[t]{2}{*}{8} & \multirow[t]{2}{*}{ adult } & $\begin{array}{l}\text { num- } \\
\text { ber }\end{array}$ & $\begin{array}{c}19 \pm 25 \\
(4)\end{array}$ & 0 & $\begin{array}{c}8 \pm 11 \\
(5)\end{array}$ & $\begin{array}{l}2 \pm 2 \\
(3)\end{array}$ & $\begin{array}{l}2 \pm 1 \\
(2)\end{array}$ & 0 & 1 & 0 & $\begin{array}{l}1 \pm 0 \\
(4)\end{array}$ & 2 & $\begin{array}{l}1 \pm 0 \\
(3)\end{array}$ & $\begin{array}{l}3 \pm 2 \\
(5)\end{array}$ & $\begin{array}{c}10 \pm 11 \\
(2)\end{array}$ & $3 \pm 2(2)$ & $\begin{array}{l}7 \pm 8 \\
(6)\end{array}$ \\
\hline & & FO & 35 & 0 & 18 & 3 & 1 & 0 & $<1$ & 0 & 2 & 1 & 1 & 7 & 9 & 2 & 20 \\
\hline
\end{tabular}

Table 7: $\mathrm{BCl}$ and $\mathrm{BB}$ of different gender and age class of green turtles.

\begin{tabular}{|c|c|c|c|c|}
\hline & Parameter & Mean & S.D. & Replicates \\
\hline \multirow[b]{2}{*}{ Male } & $\mathrm{BCI}$ & 0.09 & 0.02 & \multirow[b]{2}{*}{32} \\
\hline & BB & 4.23 & 6.75 & \\
\hline \multirow[b]{2}{*}{ Female } & BCI & 0.1 & 0.02 & \multirow[b]{2}{*}{63} \\
\hline & BB & 1.4 & 4.14 & \\
\hline \multirow[b]{2}{*}{ Unknown Sex } & $\mathrm{BCI}$ & 0.11 & 0.02 & \multirow[b]{2}{*}{38} \\
\hline & BB & 0.65 & 1.18 & \\
\hline \multirow[b]{2}{*}{ Juvenile } & $\mathrm{BCI}$ & 0.11 & 0.02 & \multirow[b]{2}{*}{110} \\
\hline & BB & 1.84 & 4.19 & \\
\hline \multirow[b]{2}{*}{ Subadult } & $\mathrm{BCI}$ & 0.09 & 0.03 & \multirow[b]{2}{*}{20} \\
\hline & BB & 2.06 & 6.65 & \\
\hline
\end{tabular}

Table 8: Number and proportion of debris contained in each section of the digestive tract.

\begin{tabular}{|c|c|c|c|c|c|c|c|}
\hline Parameter & Esophagus & Stomach & Intestine I & Intestine Ii & Intestine Iii & Intestine Iv & Intestine V \\
\hline Number & 2 & 5 & 2 & 13 & 27 & 32 & 19 \\
\hline \% Total & 2 & 5 & 2 & 13 & 27 & 32 & 19 \\
\hline
\end{tabular}




\section{Discussion}

\section{Marine debris selection of green turtle in the nearshore waters of Northern Taiwan}

Marine debris in total, represented by four major types: plastics, ropes, Styrofoam and monofilament lines [30]. In this study, except the hatchlings, soft plastic and plastic rope were the common and either important or unimportant ingested debris. Soft plastic was the common while unimportant ingested debris of hatchling. Hard plastic and foam plastic were the common while unimportant ingested debris, mainly of male, unknown sex, hatchling and juvenile turtles. Unidentified material was the common while unimportant ingested debris, mainly of juvenile and adult turtles. Thus, different gender and age class might select ingest different debris types, the major ingested types were similar. Overall, soft and hard plastics, plastic rope, foam plastic and unidentified material were the major ingested debris of green turtles in Northern Taiwan. Plastic ropes includes mainly the fishing lines such as monofilaments rope, and synthetic 'soft bait' lures rope for wrap up bags, etc. They are the marine debris originate mainly from the fishing vessels $[4,8,31]$. Results of this study suggested that the marine debris is a major source of ingested debris in the nearshore waters of Taiwan. High fishing activities may responsible for this result. In addition, plastic rope can also entangle marine debris [20], or macroalgae [2] which also ingest by accident. Both soft and hard plastic were either the common or most common ingested debris. Soft plastics (i.e. synthetic debris) include sheetlike plastic, single-use plastics such as food packaging and bags. Hard plastic includes plastic cap, piece of plastic bottles etc. $[1,6,8,32]$. These debris mainly derived from land sources of high human activities or dumpsters, and transport to the sea as garbage. Sea turtles feed on soft and hard plastics because they mistake for the natural foods $[3,13,14,33]$. Plastic may also become the base for the growth of plankton or algae $[13,34]$, results in the ingestion by accident [2]. Unidentified material was the common while unimportant ingested debris for green turtles. Green turtle is an omnivorous feeder, they are less selective compare to the carnivorous sea turtles [2,23]. Thus, it may include unidentified material as the common ingested debris. The statistical analysis showed that the green turtles ingested least amount of cotton thread and rubber. These two debris may be least expose to the animal. Turtles were found ingested less amount of metal, textile, cotton thread, and rubber than the major ingested debris such as soft and hard plastics, plastic rope and foam plastic. It is possible that these materials were occasionally encountered by the green turtles.

\section{Marine debris color ingested by green turtles}

Except the male turtles, white was the common and most important ingested color debris. Except the hatchling turtles, transparent was the important ingested color and except female and hatchling, mixture was the important ingested color debris. Thus, white, transparent and mixture were the major ingested color debris. Turtles ingested less purple and red debris than white and transparent debris. It is possible that the encounter of these color debris was less frequent than the major ingested color. Again, green turtles of different gender and age class might select ingest different color. However, the major ingested types were similar. Overall, white, transparent and mixture were the common ingested color for gre White and transparent seem the most widespread colors of floating bags, thus the highest frequent ingested debris $[4,5,11,19]$. The other possibility is that sea turtles can distinguish colors [35] and prefer certain colors when feeding [23]. Sea turtles in the nearshore shallow waters have stronger color selection than the ocean species. A large part of the nearshore species' diets is jellyfish or cephalopods [36]. They may thus mistake white and transparent debris for food and actively ingest them [3,4,10,23]. Other colors were also commonly ingested by sea turtle may relate to the mixture of these colors with the predominant colors or encrusted by natural preys $[10,12]$. Turtles select ingest mixture color may be due to the mixture of marine debris with the food they ingested

\section{Marine debris selection changes with the age classes}

This study found that the major ingested debris changed with the age class. For the hatchlings, soft and hard plastic was either the common or most common and either the important or unimportant ingested debris. For the juvenile turtles, soft plastic, plastic rope, foam plastic and unidentified material was the common or most common and either the important or unimportant ingested debris. For the subadult turtles, plastic rope and foam plastic were the common while unimportant ingested debris. For the adult turtles, plastic rope and unidentified material were the common or most common and either the important or unimportant ingested debris. Thus, it is clear that juvenile turtles ingested more debris types than the other age classes. However, there was no trend of change in color debris ingested among different age classes. Ingestion can occur at all stages of a sea turtle's lifecycle; however, it appears to be most frequent in juvenile and pelagic stages $[12,17,23]$. Ocean plastics are a combination of floating plastics (subject to wind stress) and neutrally buoyant plastics in the mixed layer [12,20,23]. Oceanic life-stage turtles were significantly more likely to ingest debris (plastic and proportionally more sheets and line) than the neritic life-stage turtles $[17,20,28]$. Juvenile turtles experienced the shift of foraging habitat from pelagic oceanic to benthic neritic habitats. Thus, they foraged more debris type than the other age classes [37]. Relationship between BB and ingested debris weight and between BCI and body size (SCI) of different gender and age class Sea turtle increase debris ingestion as they grow $[3,15,33$. Sea turtles, however, may decrease debris ingestion through naivety and/or ontogenetic shifts in diet or decrease the chance of contact marine debris by shift their habitat with age [28,37]. In this study, except the body burden (BB) of female turtle increased slightly with the body size, no relationship was found between BB and body size and between BCI and number of debris ingested in all 
genders and age classes. It is possible that the major size range of this study was too narrow; $75 \%$ was juvenile (35 to $55 \mathrm{~cm}$ in SCL). Thus, no significant relationship can be detected.

\section{Distribution of marine debris in different section of the intestine}

This study found that $78 \%$ of the ingested debris was in the large intestine (section III to $\mathrm{V}$ in Table 8). Similar results were found in the other studies [9,28,34]. Most turtles consumed debris several weeks to months prior to egestion or be found $[28,34]$. Marine debris thus can affect sea turtles through dietary dilution as it passes throughout the digestive tract and is ultimately excreted $[4,9]$. Marine debris might remain in the digestive tract for several weeks or longer in this study. The above arguments indicate that the ingestion of anthropogenic debris does not kill sea turtles in Taiwan directly, but a sublethal effect cannot be ruled out $[28,38]$. More research on this topic is warranted if we wish to understand this problem and find a proper solution to it.

\section{Conservation implications}

Anthropogenic debris is a "tragedy of the commons" that has become a major threat to the marine ecosystem $[1,2,39]$. The recent prevalence of the novel coronavirus (SARS-CoV-2) is expected to substantially increase the numbers of disposal facial masks being used; this will in turn increase the amount of plastic and microplastic in the ocean in the near future. It is thus important to determine how debris, especially plastic pollutants, affect the health of coastal environment. Sea turtles are a flagship animal, and thus the study of the marine debris they ingest can reflect the detrimental impact to the marine fauna in the region. We can also use the flagship character of sea turtles to conduct marine environment awareness campaigns to reduce the input of debris into the marine environment. There are not many studies on marine debris ingestion by sea turtles in Asia [40], and this is the first such study to be carried out in Taiwan. Taiwan has a population of approximate 23 million people concentrated in a few major cities, most of which are located near the coastal areas. Thus, the anthropogenic debris that leak into nearshore waters may not directly cause mortality in Taiwan's sea turtles, but it can still kill these organisms over time. It is important to identify such threats to the nearshore marine environment so that proper management strategies can be carried out to combat them. The other solution to the plastic problem is to transition straight economy into a circulatory economy by shifting "from a produce, use, dispose approach to a design, use, re-design/re-use approach" [39-44]. This can not only decrease the amount of trash in the environment, recycle them in a smart way, but enhance the relevant industries. For example, a high-tech company in Taiwan; LITEON Inc. has turned foam Styrofoam boxes into laptop mouses and keyboards, and turned plastic bottles into T-shirts in recent years (Personal communication). Even though they only demonstrate the ability to convert the trash into useful tools without become part of the business. They do, however, open a road to find "gold mine in the trash".

\section{Acknowledgements}

The authors thank Ms. N-J Chou, Ms. C-H Chen, Ms. I-T Chan, J-H Jen, and the volunteers in the research teams for their assistance with the field work. This project was partly supported by a grant from the 2019 and 2020 Rescue of Stranding and Bycatch sea turtles in Northern Taiwan (\#108-C-2 and 109-C-12) from the Marine Conservation Council to I-J. Cheng.

\section{Conflict of Interest}

No conflict of interest.

\section{References}

1. Campani T, Baini M, Giannetti M, Cancelli F, Mancusi C, et al. (2013) Presence of plastic debris in loggerhead turtle stranded along the Tuscany coasts of the Pelagos Sanctuary for Mediterranean Marine Mammals (Italy). Marine Pollution Bulletin 74(1): 225-230.

2. Colferai AS, Silva-Filho RP, Martins AM, Bugoni L (2017) Distribution pattern of anthropogenic marine debris along the gastrointestinal tract of green turtles (Chelonia mydas) as implications for rehabilitation. Marine Pollution Bulletin 119(1): 231-237.

3. Domenech F, Aznar FJ, Raga JA, Tomás J (2019) Two decades of monitoring in marine debris ingestion in loggerhead sea turtle, Caretta caretta, from the western Mediterranean. Marine Pollution Bulletin 244: 367-378.

4. Yaghmour F, Bousi MA, Whittington-Jones B, Pereira J, García-Nuñez S, et al. (2018) Marine debris ingestion of green sea turtles, Chelonia mydas, (Linnaeus, 1758) from the eastern coast of the United Arab Emirates. Marine Pollution Bulletin 135: 55-61.

5. Camedda A, Marra S, Matiddi M, Massaro G, Coppa S, et al. (2014) Interaction between loggerhead sea turtles (Caretta caretta) and marine litter in Sardinia (Western Mediterranean Sea). Marine Environment Research 100: 25-32.

6. Leite AS, Santos LL, Costa Y, Hatje V (2014) Influence of proximity to an urban center in the pattern of contamination by marine debris. Marine Pollution Bulletin 81: 242-247.

7. Vegter AC, Barletta M, Beck C, Borrero J, Burton H, et al. (2014) Global research priorities to mitigate plastic pollution impacts on marine wildlife. Endangered Species Research 25: 225-247.

8. Godoy DA, Stockin KA (2018) Anthropogenic impacts on green turtles Chelonia mydas in New Zealand. Endangered Species Research 37: 1-9.

9. Pham CK, Rodríguez Y, Dauph A, Carriço R, Frias JPGL, et al. (2017) Plastic ingestion in oceanic-stage loggerhead sea turtles (Caretta caretta) off the North Atlantic subtropical gyre. Marine Pollution Bulletin 121(1-2): 222-229.

10. Casale P, Freggi D, Paduano V, Oliverio M (2016) Biases and best approaches for assessing debris ingestion in sea turtles, with a case study in the Mediterranean. Marine Pollution Bulletin 110: 238-249.

11. Nelms SE, Duncan EM, Broderick AC, Galloway TS, Godfrey MH, et al. (2016) Reviews. Plastic and marine turtles: a review and call for research. ICES Journal of Marine Science 73(2): 165-181.

12. Schuyler QA, Wilcox C, Townsend KA, Wedemeyer-Strombel KR, Balazs $\mathrm{GH}$, et al. (2016) Risk analysis reveals global hotspots for marine debris ingestion by sea turtles. Global Change Biology 22(2): 567-576.

13. Abreo NGS, Macusi ED, Blatchley DD, Cuenca GC (2016) Ingestion of Marine Plastic Debris by Green Turtle (Chelonia mydas) in Davao Gulf, Mindanao, Philippines. Philippine Journal of Science 145(1): 17-23. 
14. Hoarau L, Ainley L, Jean C, Ciccione S (2014) Ingestion and defecation of marine debris by loggerhead sea turtles, Caretta caretta, from bycatches in the South-West Indian Ocean. Marine Pollution Bulletin 84(12): 90-96.

15. Lynch JM (2018) Quantities of marine debris ingested by sea turtles: global metaanalysis highlights need for standardized data reporting methods and reveals relative risk. Environment Science Technology 52(21): 12026-12038

16. Santos RG, Andrades R, Boldrini MA, Martins AS (2015) Debris ingestion by juvenile marine turtles: An underestimated problem. Marine Pollution Bulletin 93(1-2): 37-43.

17. Wilcox C, Puckridge M, Schuyler QA, Townsend K, Hardesty BD (2018) A quantitative analysis linking sea turtle mortality and plastic debris ingestion. Science Report 8, 12536.

18. Isangedighi IA, David GS, Obot OI (2018) Plastic waste in the aquatic environment: impacts and management. Cites 2:1-31.

19. Rizzia M, Rodrigues FL, Medeiros L, Ortega I, Rodrigues L, et al. (2019) Ingestion of plastic marine litter by sea turtles in southern Brazil: abundance, characteristics and potential selectivity. Marine Pollution Bulletin 140: 536-548.

20. Vélez-Rubio GM, Teryda N, Asaroff PE, Estrades A, Rodriguez D, et al. (2018) Differential impact of marine debris ingestion during ontogenetic dietary shift of green turtles in Uruguayan waters. Marine Pollution Bulletin 127: 603-611.

21. Santos RG, Andrades R, Demetrio GR, Kuwai GM, Sobral MF, et al. (2020) Exploring plastic-induced satiety in foraging green turtles. Environment Pollution 265: 114918

22. García-Besné G, Valdespino C, Rendón-von Osten J (2015) Comparison of organochlorine pesticides and PCB residues among hawksbill (Eretmochelys imbricata) and green (Chelonia mydas) turtles in the Yucatan Peninsula and their maternal transfer. Marine Pollution Bulletin 91(1): 139-148.

23. Schuyler Q Hardesty BD, Wilcox C, Townsend K (2013) Global Analysis of Anthropogenic Debris Ingestion by Sea Turtles. Conservation Biology 28(1): 129-139.

24. Wyneken J (2001) The Anatomy of Sea Turtles. NOAA Tech. Memorandum NMFS-SEFSC-470, p. 172.

25. Ferreira B, Garcia M, Jupp BP, Al-Kiyumi A (2006) Diet of the Green Turtle (Chelonia mydas) at Ra's Al Hadd, Sultanate of Oman. Chelonian Conservation Biology 5(1): 141-146.

26. von Brandis RG, Mortimer JA, Reilly BK, van Soest RWN, Branch M (2014) Diet Composition of Hawksbill Turtles (Eretmochelys imbricata) in the Republic of Seychelles. Western Indian Ocean. Journal of Marine Science 13(1): 81-91.

27. Keller JM, Pugh R, Becker PR (2014) Biological and Environmental Monitoring and Archival of Sea Turtle Tissues (BEMAST): rationale, protocols, and initial collections of banked sea turtle tissues. In: U.S. Department of Commerce (Ed.), NIST Internal Report 7996, Gaithersburg, MD.

28. Clukey KE, Lepczyk CA, Balazs GH, Work TM, Lynch JM (2017) Investigation of plastic debris ingestion by four species of sea turtles collected as bycatch in pelagic Pacific longline fisheries. Marine Pollution Bulletin 120: 117-125.

29. Sokal RR, Rohlf FJ (1982) Biometry, 2nd Ed. W.H. Freeman and Co., San Francisco, California.

30. Lazar B, Gracăn R (2011) Ingestion of marine debris by loggerhead sea turtles, Caretta caretta, in the Adriatic Sea. Marine Pollution Bulletin 62(1): 43-47.

31. Derraik JGB (2002) The pollution of the marine environment by plastic debris: a review. Marine Pollution Bulletin 44: 842-852.

32. Wedemeyer-Strombel KR, Balazs GH, Johnson JB, Peterson TD, Wicksten MK, et al. (2015) High frequency of occurrence of anthropogenic debris ingestion by sea turtles in the North Pacific Ocean. Marine Biology.

33. Schuyler Q, Hardesty BD, Wilcox C, Townsend K (2012) To Eat or Not to Eat? Debris Selectivity by Marine Turtles. PLOS ONE 7(7): e40884.

34. Carman VG, Acha EM, Maxwell SM, Albareda D, Campagna C, et al. (2014) Young green turtles, Chelonia mydas, exposed to plastic in a frontal area of the SW Atlantic. Marine Pollution Bulletin 78(1-2): 56-62.

35. Fritsches KA, Warrant EJ (2013) Chap. 2. Vision. Wyneken J, Lohmann KJ, Musick JA (eds.) The Biology of Sea Turtles, vol. III. Pp. 31 - 58.

36. Narazaki T, Sato K, Abernathy KJ, Marshall GJ, Miyazaki N (2013) loggerhead turtles (Caretta caretta) use vision to forage on gelatinous prey in mid-water. PLOS ONE 8(6): e66043.

37. Duncan EM, Arrowsmith JA, Bain CE, Bowdery H, Broderick AC, et al. (2019) Diet-related selectivity of macroplastic ingestion in green turtle (Chelonic mydas) in the eastern Mediterranean. Science Report 9(1): 11581.

38. Schuyler QA, Wilcox C, Townsend K, Hardesty BD, Marshall NJ (2014) Mistaken identity? Visual similarities of marine debris to natural prey items of sea turtles. Behavior Physiology Ecology 14.

39. Vince J, Hardesty BD (2018) Governance solutions to the tragedy of the commons that marine plastics have become. Frontal Marine Science 5: 214.

40. Ng CKY, Ang PO, Russell DJ, Balazs GH, Murphy MB (2016) Marine macrophytes and plastics consumed by green turtles (Chelonia mydas) in Hong Kong, South China Sea region. Chelonian Conserv. Biol. 15(2), 289-292.

41. Bugoni L, Petry MV (2001) Marine Debris and Human Impacts on Sea Turtles in Southern Brazil. Marine Pollution Bulletin 42(12): 1330-1334.

42. Carson HS, Lamson M, Nakashima D, Toloumu D, Hafner J, et al. (2013) Tracking the sources and sinks of local marine debris in Hawaii. Marine Environment Research 84: 76-83.

43. de Carvalho RH, Lacerda PD, Mendes S da S, Barbosa BC, Paschoalini M, et al. (2015) Marine debris ingestion by sea turtles (Testudines) on the Brazilian coast: an underestimated threat? Marine Pollution Bulletin 101(1):746-749.

44. Fukuoka T, Yamane M, Kinoshita C, Narazaki T, Marshall GJ, et al. (2016) The feeding habit of sea turtle marine debris influences their reaction to artificial marine debris. PLOS ONE 6:28015. 